\title{
DNA-Based Nanostructures: Changes of Mechanical Properties of DNA upon Ligand Binding
}

\author{
Yury Nechipurenko ${ }^{1}$, Sergey Grokhovsky ${ }^{1}$, Georgy Gursky ${ }^{1}$, \\ Dmitry Nechipurenko ${ }^{2}$, and Robert Polozov ${ }^{3}$
}

\begin{abstract}
The formation of DNA-based nanostructures involves the binding of different kinds of ligands to DNA as well as the interaction of DNA molecules with each other. Complex formation between ligand and DNA can alter physicochemical properties of the DNA molecule. In the present work, the accessibility of DNAligand complexes to cleavage by DNase I are considered, and the exact algorithms for analysis of diagrams of DNase I footprinting for ligand-DNA complexes are obtained. Changes of mechanical properties of the DNA upon ligand binding are also demonstrated by the cleavage patterns generated upon ultrasound irradiation of cis-platin-DNA complexes. Propagation of the mechanical perturbations along DNA in the presence of bound ligands is considered in terms of a string model with a heterogeneity corresponding to the position of a bound ligand on DNA. This model can reproduce qualitatively the cleavage patterns obtained upon ultrasound irradiation of cis-platin-DNA complexes.
\end{abstract}

Keywords DNA-ligand binding, DNA-based nanostructures, quantitative analysis of footprinting diagram, ultrasound DNA cleavage, string model, DNA tensegrity

\section{Introduction}

Two approaches are developed for the formation of DNA-based nanostructures. In the context of the first approach complicated interactions in the living cell are modeled by more simple interactions between DNA and synthetic ligands. For

\footnotetext{
${ }^{1}$ V. A. Engelhardt Institute of Molecular Biology, Russian Academy of Sciences, Vavilov Str. 32, Moscow, 119991, Russia

${ }^{2}$ Department of Physics, Moscow State University, Leninskie Gory, Moscow, 119992, Russia

${ }^{3}$ Institute for Theoretical and Experimental Biophysics, Moscow Region, Instituskaya Str. 3, Puschino, 142290, Russia
}

*To whom correspondence should be addressed. E-mail: nech@eimb.ru 
example, studies on the binding of various antibiotics and drugs to DNA and the synthesis of new sequence-specific DNA-binding-ligands may help to understand more complicated interactions underlying the recognition of specific target DNA sites by the transcription factors. ${ }^{1}$ The second approach implies the formation of new artificial structures stabilized by interaction between single-stranded DNA segments and complementary oligonucleotides or structures formed when DNA molecules form associates upon ligand binding. ${ }^{2,3}$

Models for a quantitative description of DNA-ligand binding have been developed in several works. ${ }^{4,9}$ A number of natural and artificial compounds bind to DNA and change its properties. In the first part of this communication we demonstrate that binding of the dimeric analogue of the antibiotic netropsin changes the accessibility of DNA for cleavage by DNase I. In the second part, we describe a model which allows us to interpret the observed cleavage patterns generated upon ultrasound irradiation of cis-platin - DNA complexes.

\section{Quantitative Analysis of Footprinting Diagrams}

Modern experimental techniques developed in the past years such as different variants of footprinting allow to determine the sites of predominant location of a ligand bound on a DNA fragment with a known sequence of base pairs. The method of footprinting is based on the fact that DNA regions occupied by a ligand are inaccessible for cleavage by DNase I or another reagent capable of hydrolyzing the sugar-phosphate backbone of DNA. However, analysis methods for footprinting diagrams, which enable one to evaluate the probabilities of ligand binding to different DNA regions, have so far only been developed insufficiently.

Here, we briefly describe the algorithm that allows to calculate the probabilities of binding a ligand with the binding sites on a DNA fragment with a known sequence of base pairs. ${ }^{6,9}$ A statistical mechanics approach allows the most complete characterization of the system and a clear representation of the effects related to its statistical properties. This algorithm can be used for the quantitative analysis of diagrams of DNase footprinting for the complexes of the dimeric analogue of the antitumor antibiotic netropsin, which exhibits a high binding selectivity. ${ }^{9}$ This antibiotic binds in the minor DNA groove to runs of four and five AT base pairs. ${ }^{10,11}$ An obvious way to increase the selectivity of binding of these compounds is to synthesize dimeric (oligomeric) analogues, in which two or more netropsin fragments are bound covalently. ${ }^{112}$ This group of compounds includes Pt-bis-netropsin, in which two netropsin fragments are covalently linked via the cis-diaminoplatinum group. ${ }^{13}$ It was shown earlier that Pt-bis-netropsin forms three types of complexes with DNA, ${ }^{13,16}$ but only one of them dominates under the experimental conditions used. 


\subsection{The Thermodynamical Model of Adsorption}

Consider a solution at constant temperature and pressure which contains DNA molecules with adsorbed ligands and free ligand molecules. The DNA molecule represents a linear polymer consisting of $N$ units (nucleotide pairs). Upon binding, the ligand occupies $L$ units of the polymer, which become inaccessible for binding with other ligand molecules. Henceforth we refer to these $L$ units as "the binding site". Assume that the ligand cannot protrude beyond DNA ends. Let us number the units of the polymer from 1 to $N$, thus the first binding site is the site including $L$ first units; the second binding site begins with the second unit and ends with the unit $L+1$, etc. By $K(i)$ we designate the constant of ligand binding with i-th binding site, which begins with the unit / and ends with the unit $i+L-1$.

All thermodynamical characteristics of the system can be calculated if the expression for the great partition function $\Xi_{N}$ is known. The latter can be calculated using the method of recurrent equations. Recurrent equations for describing noncooperative binding of extended ligands on a homo- and heteropolymer were obtained previously. ${ }^{6,8}$ In the case of noncooperative binding, the recurrent equation will be as follows:

$$
\Xi_{N}=\Xi_{N-1}+K(N-L+1) \cdot m \cdot \Xi_{N-\mathrm{L}}
$$

with boundary condition

$$
\Xi_{N}=1 \text { if } i<L, \Xi_{L}=K(1) \cdot m+1
$$

where $K(i)$ is the constant of binding of the ligand with the $i$-th binding site and $m$ is the free ligand concentration in solution. In case of specific binding on the heteropolymer, $K(i)$ depends on the nucleotide sequence in the DNA site occupied by the ligand. The probability, $P(i)$, that the $i$-th site of DNA is occupied by the ligand can be calculated using the standard equation (see Nechipurenko and $\operatorname{Gursky}^{8}$ ):

$$
P(i)=\frac{\partial \ln \Xi_{N}}{\partial \ln [K(i) \cdot m]} .
$$

\section{$2.2 \quad$ PT-Bis-Netropsin Binding with DNA}

The curves of titration of poly(dA)-poly(dT) with Pt-bis-netropsin show that the size, L, of the binding site for Pt-bis-netropsin is equal to eight base pairs. ${ }^{14,15}$ The probability $P(i)$ can be calculated using recurrent Eqs. (1) and (3), if the set of constants $K(i)(1<=i<=N-L+1)$ is known. The binding constant $K(i)$ depends on the contribution to the binding energy of both specific and nonspecific (i.e., independent of the nucleotide sequence) interactions between the ligand and DNA. To calculate the 


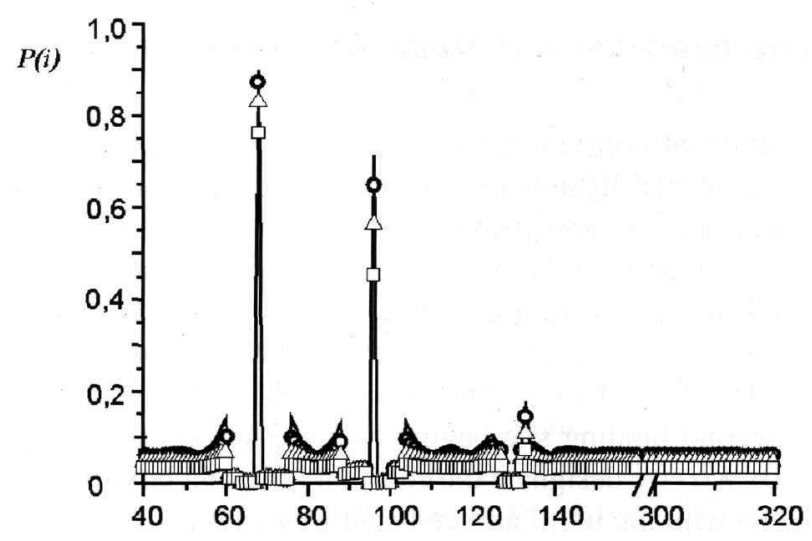

Fig. 1 Dependence of the probability of ligand binding to the i-th binding site of DNA, $P(i)$, on the base pair number, $i$. Curves were calculated for the following concentrations of free ligand in solution: $0.125 \mu \mathrm{M}(\bullet), 0.25 \mu \mathrm{M}(\mathrm{A}), 0.5 \mu \mathrm{M}(\mathrm{O})$, and $1 \mu \mathrm{M}$ (solid line)

profiles of distribution of the ligand bound on a DNA fragment TV base pairs long, it is necessary to determine the constants of binding of the ligand with $N-L+1$ overlapping binding sites on the DNA fragment with the specified nucleotide sequence. The constants $K(i)$ can be estimated based on the isotherms of adsorption of the ligand on DNA oligomers. The interaction of the ligand with weaker binding sites can be characterized using a certain binding constant $K_{A V}$, which is averaged with respect to all "weak" binding sites. In our calculations, we used the value $K_{A V}=5 \cdot 10^{5} \mathrm{M}^{-1}$. The use of a smaller $K_{A V}$ value only slightly affects the relative positions of the maxima and minima on the profiles of distribution of the ligand bound on the DNA fragment.

Figure 1 shows calculated profiles of distribution of Pt-bis-netropsin bound on a DNA fragment with known sequence 15 for different ligand concentrations.

In our calculations we used the experimental values of constants of binding of Pt-bis-netropsin with DNA regions with three strongest sites $\left(4-10^{7} \mathrm{M}^{-1}, 110^{7} \mathrm{M}^{-1}\right.$ and $\left.1.10^{6} \mathrm{M}^{-1}\right)$. The $P(i)$ values directly correlated with information obtained using the footprinting technique (see Yu et al. ${ }^{9}$ for details).

\section{The Propagation of Mechanical Perturbations along the DNA Molecule}

Protein-DNA interactions can induce DNA bending and twisting and might be responsible for a local change in electric and mechanical properties of a DNA molecule. In the last decade new approaches have been developed for studies of mechanical properties of a single DNA molecule. ${ }^{1718}$ Studies on the effect of mechanical perturbations induced in DNA upon ultrasonic irradiation in solution 
may also provide information on the mechanical properties of DNA and its complexes with regulatory proteins and anticancer drugs. Recent observations show that the efficiency of DNA breakage upon ultrasound irradiation depends on the nucleotide sequence near the cutting site. ${ }^{19}$ DNA breaks occur most frequently at 5'-CG-3' steps. The interaction of DNA with cis-platin greatly increases the probability of DNA cutting by ultrasound in the close proximity of a bound cis-platin molecule on DNA.

Here, we propose a simple model for the description of the propagation of mechanical disturbances along the DNA molecule. The model can be used to describe some of the recent experimental observations on effects of ultrasound irradiation upon DNA.

\subsection{Ultrasonic Irradiation of DNA-Ligand Complexes}

The effects of ultrasonic irradiation are based on the cavitation phenomenon: tiny bubbles are produced in solution and their collapses initiate microjets with a speed of about $100 \mathrm{~m} / \mathrm{sec}^{20}$ It is assumed that those jets are capable of breaking the sugarphosphate backbone of a DNA molecule. The sizes of the bubbles and the jet diameters have recently been estimated experimentally. ${ }^{21}$ The typical jet size is approximately $300 \mathrm{~nm}$, a value which greatly exceeds the diameter of a DNA molecule (2nm). That is why DNA fragments containing at least several hundreds of nucleotide pairs might be considered to be one-dimensional structures.

\subsection{String Model of a DNA Molecule}

The dynamical features of a DNA fragment are determined by both electrical and mechanical properties of the molecule. Repulsion between phosphate groups creates a tension within a single DNA molecule which might be compensated by the strain of valence and non-valence bonds, creating a total tension of the molecule. Thus, the DNA molecule might be considered a self-stressed mechanical construction (a tensegrity system). ${ }^{22}$ Tensegrity plays a fundamental role in many biological processes. $^{23}$ Both tensegrity and one-dimensionality make it reasonable to model the DNA fragment as a string in order to study the propagation of mechanical perturbations along the DNA.

The propagation of perturbation along the string is determined by the wave equation with the initial and boundary conditions which will be formulated as follows:

$$
\frac{\partial^{2} U}{\partial t^{2}}=\frac{F}{\rho(x)} \frac{\partial^{2} U}{\partial x^{2}}+g(x, t)
$$


where $U(x, t)$ is the transverse displacement of a string's element with the coordinate $x$ at time interval $t ; F$ is the overall tension of the string (the appropriate estimate of $F$ for a DNA molecule is about $10 \mathrm{pN}) ;{ }^{22} p(x)$ is the linear density of the string (approximately $3-10^{-15} \mathrm{~kg} / \mathrm{m}$ for DNA); $g(x, t)$ - is the external force (which acts on the string at position $x$ and time $t$ ) divided by the linear density $p(x)$.

$C=\sqrt{ }[F / \rho(x)]$ gives the speed of propagation of transverse perturbations along the string. The estimated value of this parameter is about $100 \mathrm{~m} / \mathrm{sec}$.

We considered the problem of propagation of the mechanical impulse along the string provided the linear density of the string involves a step-like heterogeneity. The external force has been chosen to be of exponential form:

$g(x, t)=[A / \rho(x)] \exp \left[-a \cdot\left(t^{2}+2 \cdot\left(x-x_{o}\right) / b\right)\right]$, here $x_{o}$ is the centre of the application of external force; $A, a, b$ are parameters.

The numerical values have been chosen to make the time span of the force action shorter than the time needed for the propagation of a disturbance along the string. This mode of force action might correspond to the interaction of DNA with cavitational microjets in the regions of high velocity gradients.

The numerical values of parameters used in calculations are as follows: the string's length $/=100$, so that $x$ varies in the interval: $0<x<100 ; A=600 ; a=1$; $b=30 ; C^{2}=F / \rho(x)=10$. The linear density of the string is assumed to be a step function: in a small region of the string the value of density is twofold greater than in other string parts:

$$
\rho(x)=1 \text { if } 0 \leq x \leq 70 \text { and } 80 \leq x \leq 100 ; \rho(x)=2 \text { if } 70<x<80 .
$$

A step-wise change of linear density may model complexes of DNA with proteins and anticancer drugs. A typical example is the binding of cis-platin, which forms a crosslink between adjacent guanine residues on the same DNA strand and increases the mass of the corresponding DNA segment.

Equation (4) with zero displacements and zero velocities as initial conditions and free-ends of string as boundary conditions has been solved numerically. We have analyzed the time-dependence of deformation energy density. To solve the equation, the standard "cross" difference scheme has been used. The analytical grid has been chosen to have coordinate step $A x=1$ and time step $A t=0.001$ These values make the difference scheme stable. ${ }^{24}$

The calculation shows that when perturbation reaches the region of heterogeneity on a string, there is a considerable increase in the deformation energy density at the boundaries between string segments with different linear densities. This effect is attributed to the string's heavy region's dynamics: since this region has a greater inertia $(\mathrm{p}(\mathrm{x})=2)$, it can not respond to the perturbation as rapidly as the adjacent segment which is characterized by a lower linear density value $(p(x)=1)$. This is why the amplitude of string deformation increases.

Figure 2 shows the deformation energy profiles for two points in time shortly after the initial perturbation of the string occurs.

Figure 3 illustrates the string's deformation energy profile at the time $t_{3}$, when the wave reaches the density's heterogeneity. One can see that an increase of the deformation energy takes place on the boundary between "light" and "heavy" string 
segments $(x=70)$. It is significant that the effect still takes place when the initial impulse is applied directly to the region where string heterogeneity is located.

The string model clearly demonstrates that deformation energy increases at the boundary between "light" and "heavy" string segments. Such an increase might be considered as a basis for the explanation of recent observations showing that the probability of DNA breakage by ultrasound is increased at the preferred ligand binding site on DNA. ${ }^{19}$

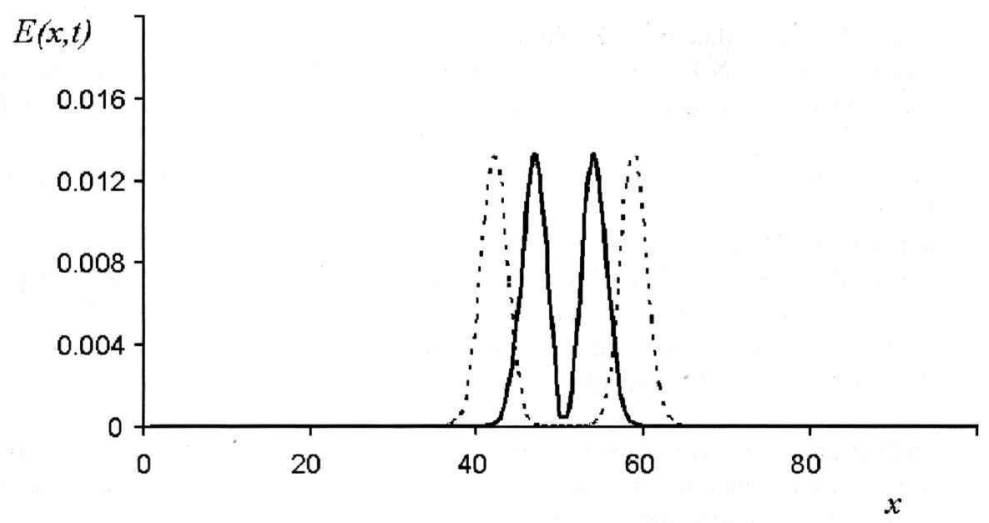

Fig. 2 The string's deformation energy profiles for two points in time shortly after the initial perturbation, $x$ - distance from the left end of the string; $E(x, t)$ - string's deformation energy density

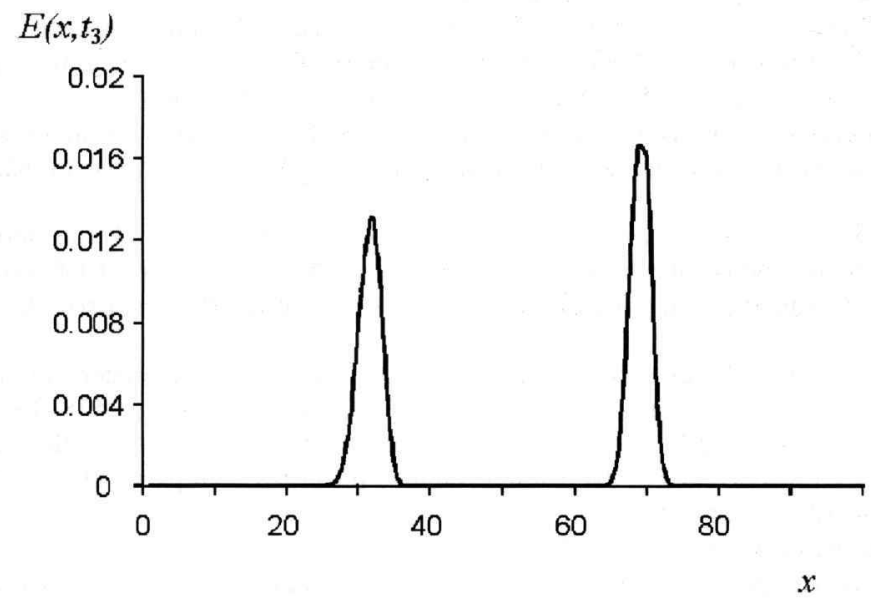

Fig. 3 The string's deformation energy profile at the moment when the perturbation wave reaches the boundary of linear density's heterogeneity, $x$ - distance from the left end of the string; $E\left(x, t_{3}\right)$ - string's deformation energy density. The deformation energy increases at the boundary of heterogeneity $(x=70)$ 
Acknowledgements We would like to thank V. F. Ryabokon, B. Jovanovic, E. A. Vorobiev, and M. V. Golovkin for helpful discussions and technical assistance. This research was supported by State Grant "Construction of new drugs for therapy and prophylaxis of antiviral diseases by methods of organic chemistry", Grant of Presidium RAN for Molecular and Cell Biology and RFBR grant 04-04-49364.

\section{References}

1. G. V. Gursky, A. S. Zasedatelev, A.L. Zhuze, A. A. Khorli., S. L. Grokhovsky, S. A. Streltsov, A. N. Surovaya, A. M. Nikitin, A. S. Krylov, V. O. Retchinsky, R. S. Beabealashvili, and B. P. Gottikh, Synthetic sequence-specific ligands, Cold Spring Harbor Symp. Quant. Biol. 47, 367-378 (1983).

2. N. C. Seeman, Structural DNA nanotechnology: an overview, Methods Mol. Biol. 303, 143166(2005).

3. M. A. Zakharov, Yu. M. Yevdokimov, S. G. Skuridin, and Yu. D. Nechipurenko, Nanotechnology based on spatially fixed ds DNA (RNA) molecules, Nanotech. Technical Proceedings of the 2005 NSTI Nanotechnology Conference and Trade Show 1, 288-291 (2005).

4. A. S. Zasedatelev, G. V. Gursky, and M. V. Vol'kenshtein, Theory of one-dimensional adsorption. I. Adsorption of small molecules on a homopolymer, Mol. Biol. (Moscow) 5, 194-198 (1971).

5. J. D. McGhee and P. H. von Hippel, Theoretical aspects of DNA-protein interactions: cooperative and non-co-operative binding of large ligands to a one-dimensional homogeneous lattice. J. Mol. Biol. 86, 469-489 (1974).

6. G. V. Gursky and A. S. Zasedatelev, Thermodynamical and stereochemical aspects of binding interactions between sequence-specific ligands and DNA, Sov. Sci. Rev. D. Physicochem. Biol. 5, 53-139 (1984).

7. Yu. D. Nechipurenko and G. V. Gursky, Cooperative effects on binding of proteins to DNA, Biophys. Chem. 24, 195-209 (1986).

8. Yu. D. Nechipurenko and G. V. Gursky, Thermodynamical models of binding ligands to nucleic acids, Biofizika. 48, 773-796 (2003).

9. Yu. D. Nechipurenko, B. Jovanovic, V. F. Riabokon, and G. V. Gursky, Quantitative methods of analysis of footprinting diagrams for the complexes formed by a ligand with a DNA fragment of known sequence, Ann. N. Y. Acad. Sci. 1048, 206-214 (2005).

10. M. L. Kopka, C. Yoon, D. Goodsell, P. Pjura, and R. E. Dickerson, The molecular origin of DNA-drug specificity in netropsin and distamycin, Proc. Natl. Acad. Sci. USA. 82, 1376-1380 (1985).

ILL. Tabernero, N. Verdaguer, M. Coll, I. Fita, G. A. van der Marel, J. H. van Boom, A. Rich, and J. Aymami, Molecular structure of the A-tract DNA dodecamer d(CGCAAATTTGCG) complexed with the minor groove binding drug netropsin, Biochemistry. 32, 8403-8410 (1993).

12. C. Bailly and J. B. Chaires, Sequence-specific DNA minor groove binders, Design and synthesis of netropsin and distamycin analogues, Bioconjug. Chem. 9, 513-538 (1998).

13. S. L. Grokhovsky and V. E. Zubarev, Sequence-specific cleavage of double-stranded DNA caused by x-ray ionization of the platinum atom in the Pt-bis-netropsin - DNA complex, Nucl. Acids Res. 19, 257-264 (1990).

14. A. N. Surovaya, G. Burckhardt, S. L. Grokhovsky, E. Birch-Hirschfeld, G. V. Gursky, and C. Zimmer, Hairpin polyamides that use parallel and antiparallel side-by-side peptide motifs in binding to DNA, J. Biomol. Struct. Dyn. 14, 595-606 (1997).

15. S. L. Grokhovsky, A. N. Surovaya, G. Burckhardt, V. F. Pismensky, B. K. Chernov, C. Zimmer, and G. V. Gursky, DNA sequence recognition by bis-linked netropsin and distamycin derivatives, FEBS Lett. 439, 346-350 (1998). 
16. A. N. Surovaya, S. L. Grokhovsky, G. Burckhardt, H. Fritzsche, C. Zimmer, and G. V. Gursky, Effect of DNA local conformation on the affinity and binding specificity of bis-netropsins to DNA, Mol. Biol. (Moscow) 36, 901-911 (2002).

17. C. Bustamante, Z. Bryant, and S. B. Smith, Ten years of tension: single-molecule DNA mechanics, Nature 421, 423^27 (2003).

18. C. Bustamante, Y. R. Chemla, N. R. Forde, and D. Izhaky, Mechanical processes in biochemistry, Ann. Rev. Biochem. 73, 705-748 (2004).

19. S. L. Grokhovsky, The specific cleavage of DNA with ultrasound, Mol. Biol. (Moscow) 40, 317-325 (2006).

20. Yu. A. Grivnin and S. P. Zubrilov, Influence of the physical properties of fluids on pulsation and destruction of non-spherical cavitational bubbles, Zh. Fiz. Khim. (Moscow) 54, 56-59 (1980).

21. J. Lee, M. Ashokkumar, S. Kentish, F. Grieser, Determination of the size distribution of sonoluminescence bubbles in a pulsed acoustic field, JACS. 127, 16810-16811 (2005).

22. D. Yu. Nechipurenko, R. V. Polozov, Yu. D. Nechipurenko, DNA as self-stressed mechanical construction, Biofizika (in press).

23. D. E. Ingber, Tensegrity I. Cell structure and hierarchical systems biology, J. Cell Sci. 116, 1157-1173(2003).

24.1. S. Berezin, N. P. Zhidkov, Metodi Vichislenii, V. 2, (Moscow, Fizmatgiz, 1960) [in Russian]. 